\title{
Stearylated octaarginine and artificial virus-like particles for transfection of siRNA into primary rat neurons
}

\author{
LARS TÖNGES, ${ }^{1,4}$ PAUL LINGOR, ${ }^{1,3,4}$ ROMAN EGLE, $^{2}$ GUNNAR P.H. DIETZ, ${ }^{1,3}$ ALFRED FAHR, ${ }^{2}$ \\ and MATHIAS BÄHR ${ }^{1,3}$ \\ ${ }^{1}$ Department of Neurology, Georg-August-University Göttingen, Faculty of Medicine, Waldweg 33, 37073 Göttingen, Germany \\ ${ }^{2}$ Department of Pharmaceutical Technology, Friedrich-Schiller-University Jena, Lessingstr. 8, 07743 Jena, Germany \\ ${ }^{3}$ DFG-Research Center for Molecular Physiology of the Brain (CMPB), Göttingen, Germany
}

\begin{abstract}
RNA interference (RNAi) provides a powerful experimental tool for sequence-specific gene silencing, allowing efficient analysis of gene function in a multitude of cell types. However, application of RNAi in primary mammalian neurons has been limited by low-transfection efficiency and considerable toxicity of conventional transfection methods. In this study, we evaluated a peptide-mediated and a polymer/lipid-based cellular delivery method for siRNA into rat primary neurons and compared the results with a commonly used liposomal transfection reagent. Stearylated octaarginine (Stearyl-R8) was used as polypeptide and artificial virus-like particles (AVPs) were used as a combined liposomal-polymeric vector, since both reagents have been previously shown to successfully transfect DNA into cell lines. Stearyl-R8 and AVPs both promoted siRNA transfection into primary hippocampal neurons via the endosomal pathway. SiRNA-mediated gene silencing could be effectively induced in primary neuron cultures. In comparison with the commonly used cationic liposome transfection agent, both novel reagents were less detrimental to cell metabolic activity. We conclude that these novel transfection methods yield performances comparable to cationic liposome-mediated transfection for siRNA, while being less cytotoxic in primary neurons. Stearyl-R8 and AVPs may therefore represent novel and more cost-efficient alternatives to conventional siRNA-transfection reagents.
\end{abstract}

Keywords: RNAi; transfection; cytotoxicity; polypeptide; virus-like particle

\section{INTRODUCTION}

RNA interference induced by introduction of siRNA has become an important research tool for targeted gene ablation in neuronal cells. However, functional studies using RNA interference depend not only on successful gene silencing, but also on unimpaired viability of the culture by the transfection reagents themselves.

A large number of commercial transfection reagents are available for the introduction of nucleic acids into cell lines. The most frequently applied method uses cationic lipidbased reagents (Maurer et al. 1999; Dalby et al. 2004). Cationic lipid-based transfection reagents efficiently deliver siRNA into cell lines without exerting major cytotoxic side

\footnotetext{
${ }^{4}$ These authors contributed equally to this work.

Reprint requests to: Paul Lingor, Department of Neurology, GeorgAugust-University Göttingen, Faculty of Medicine, S2-Laboratory, Waldweg 33, 37073 Göttingen, Germany; e-mail: plingor@gwdg.de; fax: 49-55139-8405.

Article published online ahead of print. Article and publication date are at http://www.rnajournal.org/cgi/doi/10.1261/rna.2252206.
}

effects. On the other hand, such compounds are toxic for many primary mammalian cells, e.g., primary endothelial, dendritic, or smooth muscle cells, and primary neurons (Davidson et al. 2004; Kiefer et al. 2004). Nevertheless, cationic lipid-based transfection reagents have been used for the induction of RNA interference in primary neurons when long-term survival of the culture represented a less critical issue (Krichevsky and Kosik 2002; Lingor et al. 2004).

Recently, various innovative reagents have been successfully used to transfect DNA into cell lines. Lipophilic polypeptides, such as stearylated octaarginine (StearylR8), are able to encapsulate and shuttle nucleic acids through cell membranes without the need to covalently attach the nucleic acid to the arginine-containing carrier peptide. For DNA transfection, Stearyl-R8 has proven to be a superior peptide-based transfection reagent that is at least as effective as conventional lipid-based reagents (Futaki et al. 2001a). The stearylation on the $\mathrm{N}$ terminus of the peptides increases the efficiency of transfection by facilitating the absorption of the complex to the membranes through its hydrophobic moiety. 
Consequently, new lipid-based transfection methods have been developed that use polymeric vectors combined with liposomal artificial virus-like envelopes (so-called artificial virus-like particles, AVPs). Here, nucleic acids are condensed with a potent transfection reagent (polyethyleneimine or protamine sulfate) and complexed with a liposomal envelope (artificial viral envelope, AVE) that mimics the lipid composition of natural enveloped viruses. Endosomal escape is facilitated because of additional endosomolytic properties. The use of AVEs has been shown to yield significantly improved transfection rates for DNA, for example, into primary endothelial cells (Muller et al. 2001; Nahde et al. 2001).

In our present study, both novel peptide-based (Stearyl-R8) and lipid-based (AVP) delivery methods were applied for the transfection of siRNAs into rat primary neurons and compared with a widely used liposomal transfection reagent (Lipofectamine). We evaluated the uptake mechanism and intracellular localization of siRNA, verified RNAi induction, and assessed the impairment of cell metabolic activity as a measure for cytotoxic effects.

\section{RESULTS}

\section{Intracellular localization of transfected siRNA}

The intracellular localization of siRNA after transfection is crucial for its successful function as an inductor of the RNA interference process. DNA transfection approaches depend on efficient delivery of the nucleic acid to the nucleus. For induction of RNAi, cytoplasmatic delivery of siRNA is required, because the assembly of the siRNAcontaining RISC complex occurs in the cytoplasm (Zeng and Cullen 2002; Sontheimer 2005). In this study, we used Cy3-labeled siRNA in order to visualize transfection kinetics and intracellular localization of siRNA. Fluorescent siRNA was transfected into primary neurons either in conjugation with the conventional transfection reagent Lipofectamine, the novel siRNA transfection reagents Stearyl-octaarginine (Stearyl-R8), or as artificial virus- like particles (AVPs). "Naked" siRNA applied without any transfection reagent served as control.

Except for "naked" siRNA (Fig. 1D,E), all tested transfection agents initially resulted in the formation of extracellular siRNA-containing complexes, which eventually adhered to the cell membrane and could be visualized by conventional epifluorescence. The most prominent extracellular
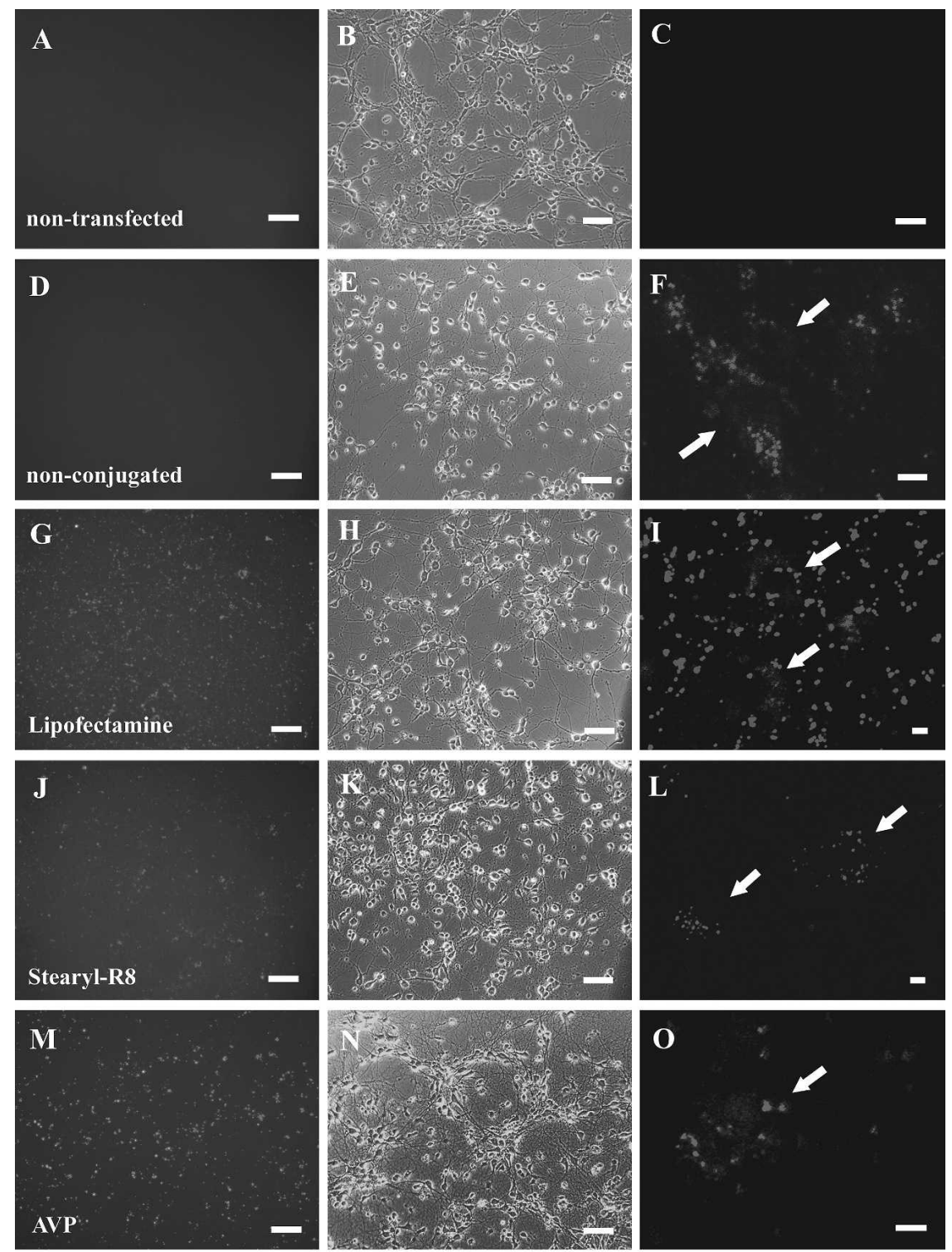

epifluorescence

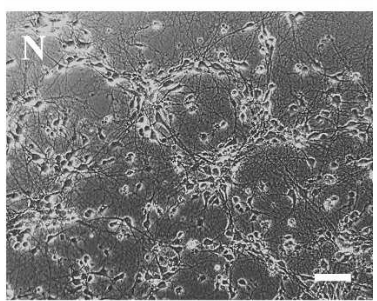

phase contrast

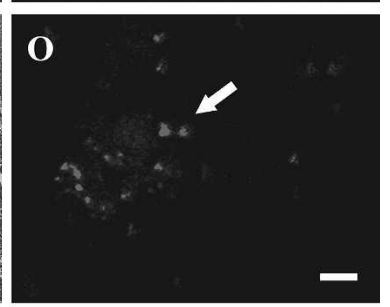

confocal
FIGURE 1. SiRNA transfection of primary hippocampal neuron cultures. Epifluorescence (left), corresponding phase contrast (middle) overview images and confocal microscopy (right) detail images of primary neuron cultures $24 \mathrm{~h}$ after transfection. Nontransfected cells are shown in $A-C$. SiRNA was applied nonconjugated $(D-F)$ or conjugated with Lipofectamine $(G-I)$, Stearyl-R8 $(J-L)$, and AVP $(M-O)$. Epifluorescence images demonstrate the distribution of siRNA conjugates in the culture with variable aggregate formation. Confocal images show the intracellular distribution of labeled siRNA within granular structures $72 \mathrm{~h}$ after transfection in greater detail. Individual cells in confocal images are labeled with arrows. Bars in epifluorescence and phase contrast images, $50 \mu \mathrm{m}$; bars in confocal images, $10 \mu \mathrm{m}$. 
liposome/siRNA aggregates were observed after application of Lipofectamine-conjugated siRNA and persisted over several days in culture (Fig. 1G,H). Extracellular aggregate formation was less prominent with Stearyl-R8- and AVPconjugated siRNA (Fig. 1J,K,M,N). Confocal images taken 72 $\mathrm{h}$ after transfection show prominent granular localization of Cy3 fluorescence next to diffuse cytoplasmatic staining (Figs. 1I,L,O). As we have previously shown (Lingor et al. 2004), application of nonconjugated "naked" siRNA to primary neurons also induced a modest granular uptake of siRNA (Fig. 1F).

Double-labeling with the lysosomal marker Lamp1 demonstrated that the granular structures containing the complexes of siRNA and transfection reagent were present in the lysosomal compartment (Fig. 2). The final intracellular distribution pattern was not markedly different between the three transfection reagents tested. Notably, the nucleus remained spared of the $\mathrm{Cy} 3$ signal in all
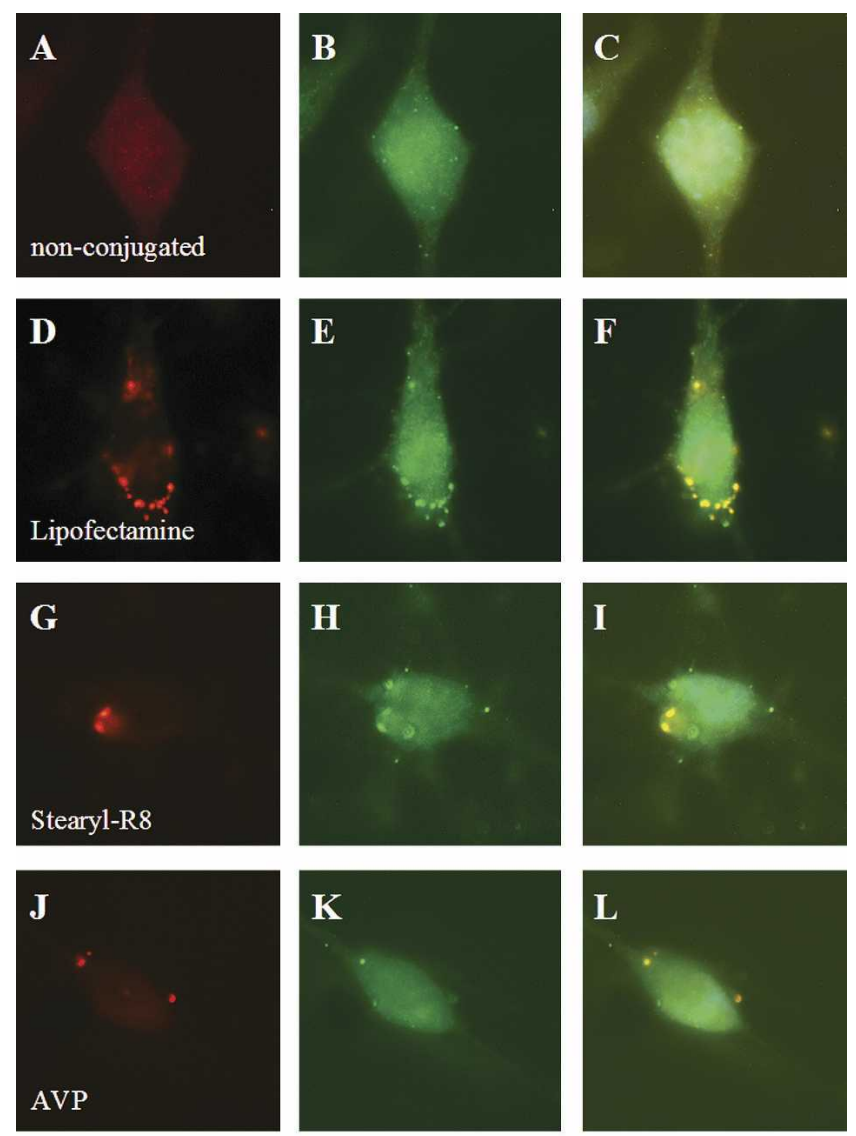

Cy3 siRNA

Lamp1

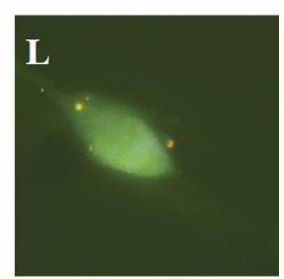

Overlay + DAPI

FIGURE 2. Intracellular localization of transfected Cy3-labeled siRNA. Transfection with Lipofectamine $(D-F)$, Stearyl-R8 $(G-I)$, and AVP $(J-L)$ results in a lysosomal localization of intracellular Cy3labeled siRNA, as demonstrated by colocalization with the lysosomal marker Lamp1 $24 \mathrm{~h}$ after transfection. Nonconjugated siRNA $(A-C)$ shows a less-intense fluorescence pattern, indicating low-transfection efficiency. transfection paradigms, as visualized by confocal images and immunostaining. All three tested transfection reagents thus mediated endocytic uptake of siRNA, which finally resulted in cytoplasmic localization of the nucleic acid.

To examine the kinetics of siRNA uptake, we performed time-lapse microscopy. Once siRNA/transfection reagent complexes adhere to the cell membrane, they can divide into multiple siRNA-containing particles, which then diminish in size and eventually decrease in fluorescence. Meanwhile, an increase of cytoplasmatic Cy3 fluorescence is observed, where RNAi could occur. Figure 3 shows this process for Stearyl-R8-transfected neurons. Similar uptake kinetics were observed using AVP-transfected siRNA (data not shown).

The transfection efficiency was determined by quantification of the percentage of Cy3-positive cells relative to all cells in culture (Table 1). The percentage of transfected cells was the lowest for nonconjugated "naked" siRNA (59 \pm $5 \%$ ), while Lipofectamine showed the highest transfection efficiency $(83 \pm 4 \%)$. Novel transfection reagents displayed slightly lower transfection efficiencies compared with Lipofectamine (Stearyl-R8 $73 \pm 2 \%$, AVP $75 \pm 4 \%$ ).

\section{Induction of RNA interference by transfected siRNA}

In order to demonstrate that conjugated siRNA remains functional, the induction of RNA interference was tested for an exogenously transfected reporter protein (EGFP) and the endogenous structure protein MAP2B.

Hippocampal neurons were transfected with an EGFPexpressing DNA plasmid by nucleofection, and EGFP expression was allowed to continue for $3 \mathrm{~d}$ in vitro (Fig. 4A). Anti-EGFP siRNA was then applied to the primary neuron cultures in conjugation with Lipofectamine (Fig. 4B), StearylR8 (Fig. 4C, Stearyl-R8:siRNA ratio of 2:1), or AVPs (Fig. 4D).

Silencing of EGFP expression was quantified by counting EGFP-positive neurons $48 \mathrm{~h}$ after siRNA application. Lipofectamine, Stearyl-R8- and AVP- effectively induced siRNA-mediated gene silencing of the EGFP reporter gene. Using the same amount of siRNA, Stearyl-R8- and AVPmediated siRNA delivery silenced EGFP expression more efficiently than Lipofectamine (Fig. 4I). Transfection of "naked" siRNA has previously been shown to have only minimal effects on EGFP expression (Lingor et al. 2004).

In order to verify the specificity of siRNA-induced gene silencing and to exclude unspecific silencing effects limited to the exogenously transfected EGFP, we targeted the endogenous MAP2 gene. After plating, neurons were transfected with anti-MAP2 siRNA on DIV2 and the downregulation was observed on DIV 9 by immunocytochemistry. Anti-EGFP siRNA served as control. All tested transfection reagents induced a down-regulation of MAP2 as visualized by decreased immunofluorescence for MAP2B, a splicing variant, which is highly expressed throughout neuronal development (Fig. 5A-N). The silencing activity in the total 

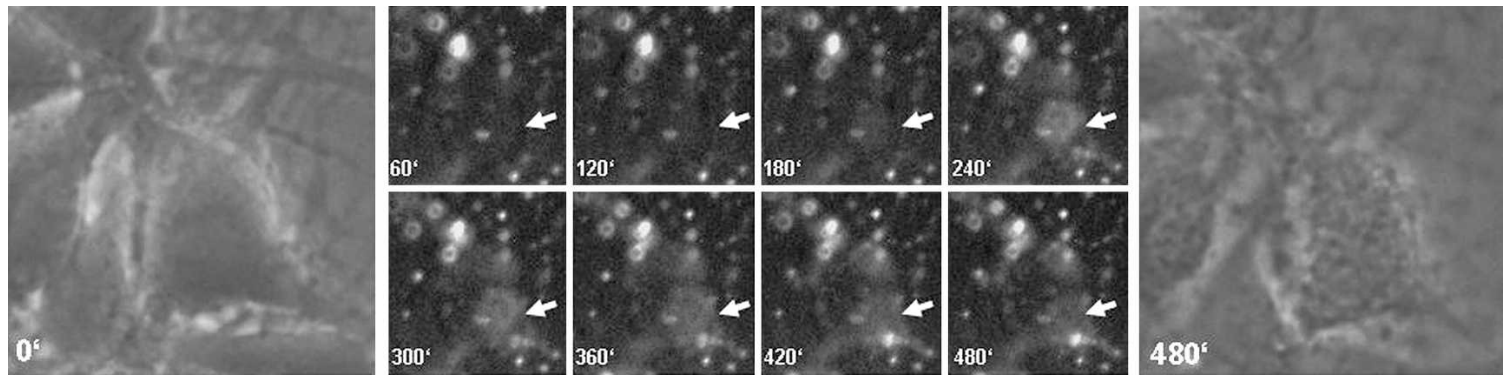

FIGURE 3. Neuronal uptake of Cy3-labeled siRNA transfected with Stearyl-R8 shown by time-lapse microscopy ( 8 h). (Left) Start of live imaging; (right) end of live imaging in phase contrast; (middle) sequential images of live microscopy taken in intervals of 60 min. Arrows indicate the same neuron throughout the time-lapse imaging. SiRNA-containing, Cy3-positive vesicles in the culture medium adhere to the cell membrane. Cellular Cy3 fluorescence representing transfected siRNA increases over time in the cell cytosol, sparing the nucleus.

culture was additionally verified by Western blots of protein lysates obtained from cultures on DIV 9 (Fig. 6).

\section{Cytotoxicity of transfection reagents for primary neuron cultures}

Next to successful induction of RNA interference, the lowest possible cytotoxic effects in primary neuron cultures were our second major criterion in the evaluation of novel siRNA transfection reagents. To evaluate cytotoxic effects in culture, we measured cell metabolic activity as a marker for functional integrity and survival of primary neurons after application of conjugated siRNA. In contrast to frequently used nuclear stains (e.g., Hoechst), quantification of mitochondrial metabolic activity has been shown to detect functional changes at an earlier stage, where nuclear morphology is yet unchanged (Vistica et al. 1991).

Lipofectamine considerably impaired cell viability, reducing the metabolic activity to $73 \%$, compared with nontransfected controls of our primary neuron culture paradigm (Fig. 7, third column) $24 \mathrm{~h}$ after transfection. SiRNA transfection using Lipofectamine additionally started inducing morphologic changes in neurites already after $12 \mathrm{~h}$ in culture (data not shown), which is in accordance with previous reports of cationic lipid toxicity (Lappalainen et al. 1994).

All tested Stearyl-R8:siRNA ratios induced no significant impairment of metabolic activity, ranging from $89 \%$ to $96 \%$ compared with the control culture (Fig. 7, fourth and

TABLE 1. Transfection efficiencies of tested transfection reagents

Transfection efficiency (percentage of

Transfection reagent Cy3-positive cells)

Nonconjugated ("naked")

$59 \pm 5 \%$

Lipofectamine

$83 \pm 4 \%$

Octaarginine (Stearyl-R8)

$73 \pm 2 \%$

Artificial virus-like particles (AVP)

$75 \pm 4 \%$ fifth column). We noted a tendency to increased toxicity with higher Stearyl-R8:siRNA ratios, which, however, remained insignificant as compared with controls. AVPmediated siRNA transfection did not show any significant impairment of metabolic activity in our culture paradigm (Fig. 7, last column).

\section{DISCUSSION}

Numerous innovative reagents for nucleic acid transfection have been developed in the past two decades. Strategies focused on peptide-mediated and polymer/lipid-based cellular DNA delivery. Here, we have applied these strategies to transfect siRNA into primary neurons and compared the efficiency to an established cationic lipid-based transfection reagent, Lipofectamine. Next to a successful delivery of siRNA to the primary cells, a main objective of our study was to determine the cytotoxic effects of the novel transfection reagents.

Futaki and coworkers have tested several oligoarginine compounds composed of four, eight, 12, or 16 arginine residues with and without stearylation for DNA transfection (Futaki et al. 2001a,b). Stearylated octaarginine has been shown to be the most efficient compound to transfect plasmid DNA into COS-7 cells, and was therefore selected for our siRNA transfection approach. We used Stearyl-R8 in a similar cation:anion charge ratio as described for the DNA transfection approach. Since the total amount of nucleic acids has to be lower for siRNA transfection approaches in order to retain specificity of gene silencing and avoid the induction of an interferon response (Sledz et al. 2003), the amount of octaarginine used was equally lower as compared with DNA transfection methods. In the case of AVPs, the same holds true. The reduced concentration required of both novel transfection reagents most likely explains the low cytotoxic side effects seen in our primary culture (Lingor et al. 2004). Rapid degradation of arginyl peptides by intracellular trypsin-like proteases could additionally account for the low cytotoxicity of the tested polypeptide (Futaki et al. 2001b). 

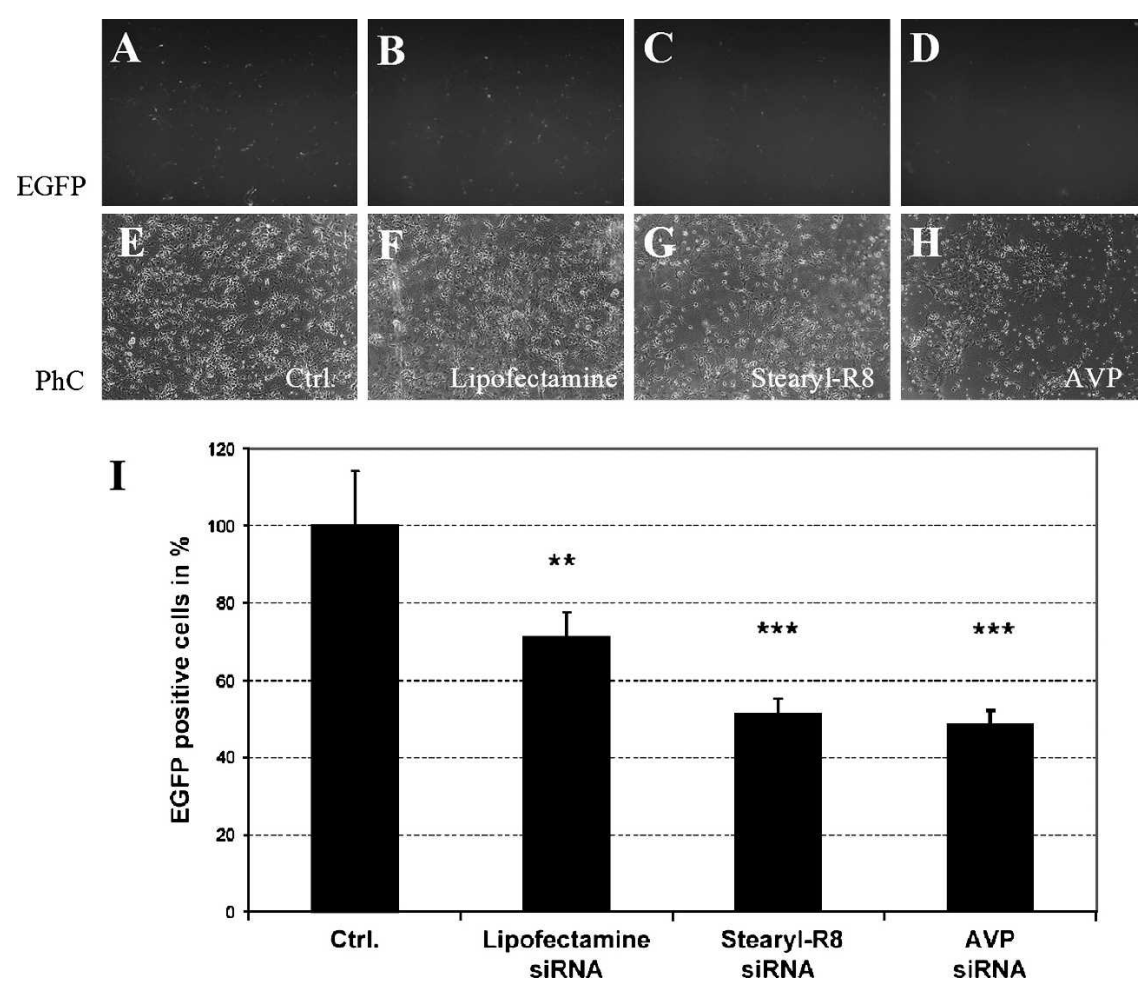

FIGURE 4. RNAi induction in primary neuronal cultures. Lipofectamine $(B)$, Stearyl-R8 $(C)$, and AVP $(D)$ effectively induce siRNA-mediated gene silencing in primary neurons previously transfected with an EGFP-expressing plasmid. EGFP-fluorescence $(A-D)$, phase contrast $(E-H)$ microscopy, and quantification of EGFP-positive cells (I) are shown.

Branched histidine and lysine polymers have been used in a similar manner for siRNA transfection approaches, demonstrating that the ability to successfully transfect DNA does not per se qualify for effective siRNA transfection (Leng et al. 2005). Other approaches used siRNA chemically coupled to the membrane permeant peptides penetratin 1 or transportan via a disulfide bond and have equally shown to mediate efficient transfection of primary neurons (Davidson et al. 2004; Muratovska and Eccles 2004). However, siRNAs have to be synthesized with a $5^{\prime}$ thiol on the sense strand, and the coupling procedure has to be performed before application, rendering this approach more elaborate and cost expensive.

Lipoplexes from anionic lipids have been previously shown to successfully transfect plasmid DNA into cell lines and primary neurons (Nahde et al. 2001; da Cruz et al. 2004; Patil et al. 2004). We now show that these so-called artificial virus-like particles are equally effective in transduction of small double-stranded RNA molecules for induction of RNA interference. Delivery systems using anionic liposomes seem to be less cytotoxic in vitro than cationic gene delivery systems (Welz et al. 2000), which we equally observed in our siRNA transfection experiments. This can be partly due to a more physiologic composition of the anionic lipids, which limits unspecific interactions with the cell surface as it is seen with cationic transfection agents (Muller et al. 2001; Nahde et al. 2001). Stearylated octaarginine, however, which is a highly basic compound, was not toxic in our experiments, which may be due to its intracellular degradation.

As shown by time-lapse- and confocal microscopy, Stearyl-R8 and AVPs formed complexes with fluorescent siRNA and resulted in formation of granule-like intracellular inclusions, which could be attributed to the lysosomal compartment. Similarly to Lipofectamine, Stearyl-R8- and AVP- thus use the endosomal uptake machinery in order to shuttle siRNA into the cytoplasm-a mechanism that has been described for most chemical transfection methods (Luo and Saltzman 2000). In the cytoplasm, less intense Cy3 fluorescence was found, indicating that $\mathrm{Cy} 3-$ labeled siRNA had been released from lysosomal compartments. The lysosomal release has been shown to be a crucial property of nucleic acid transfection reagents, as RNA degradation occurs in the cell cytoplasm (Zeng and Cullen 2002). We did not observe any nuclear translocalization of siRNA. Unlike for DNA transfection, this is not required for efficient RNAi induction. Using EGFP as reporter protein, we confirmed that siRNA transfected with Stearyl-R8 or AVPs effectively induces gene silencing in primary neurons. The silencing efficiency did not significantly differ between the three transfection methods evaluated. RNAi-induced silencing was not limited to exogenously transfected EGFP, but equally down-regulated the endogenous neuronal structure protein MAP2B, while not affecting levels of two other structure proteins $\mathrm{f}$-actin or $\beta$-tubulin.

In summary, we present two additional alternatives for the transfection of siRNA into primary mammalian neurons that could be particularly useful when only little impairment of cell viability after transfection is desired. The peptide-mediated transfection with stearylated octaarginine as well as the polymer/lipid-based cellular delivery with artificial virus-like particles both are easy to handle, as only pipet mixing of siRNA and the respective transfection reagent is necessary, and no further chemical modifications of the siRNA have to be performed. Both reagents represent cost-efficient alternatives to cationic lipid-based transfection reagents, because low amounts of transfection reagent are required.

These novel methods for siRNA delivery thus foster the use of RNAi as an experimental biological tool and in 

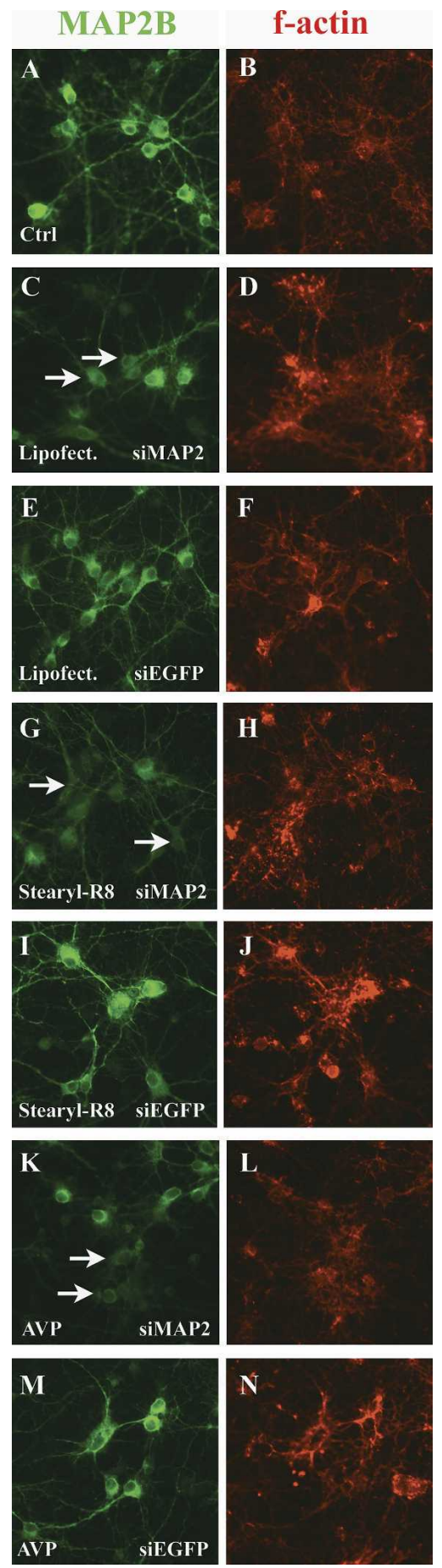

FIGURE 5. Silencing of endogenous MAP2B in hippocampal cultures. Nontransfected control cultures show a homogeneous MAP2B staining $(A)$. Counterstaining with phalloidin (indicative for f-actin) served as control for a second, nonregulated structure protein $(B)$. Cultures treated with siRNA against MAP2 show an increased number of neurons, which show a weak immunoreactivity for MAP2B $(C, G, K)$. The arrows mark exemplary neurons with most prominent down-regulation. Cultures treated with anti-EGFP siRNA show an unchanged immunoreactivity for MAP2B $(E, I, M)$. Phalloidin counterstain indicates unaltered levels of the nontargeted structure protein $\mathrm{f}$-actin $(D, F, H, J, L, N)$.

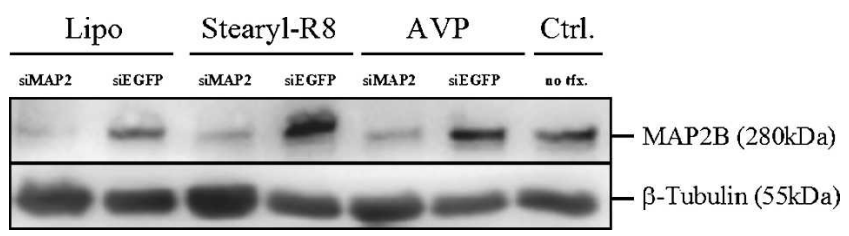

FIGURE 6. Western blots demonstrating specific down-regulation of the endogenous MAP2B protein. Anti-MAP2 siRNA transfected with Lipofectamine, Stearyl-R8, or with AVPs reduces MAP2B expression to similar levels. Control transfections using anti-EGFP siRNA do not influence MAP2B expression. All lanes were loaded with the same amount of total protein as indicated by the $\beta$-Tubulin detection.

putative therapeutic approaches for targeted inhibition of genes involved in the pathogenesis of human disease.

\section{MATERIALS AND METHODS}

\section{Primary hippocampal neuron culture}

Briefly, pregnant Wistar rats were sacrificed at embryonic day 18 by $\mathrm{CO}_{2}$ intoxication. Both hippocampi were dissected and tissue pieces were trypsinated and dissociated using a fire-polished Pasteur pipette. Cells were seeded on poly-L-ornithine/laminin (both Sigma-Aldrich)-coated glass coverslips at a density of $50,000 / \mathrm{cm}^{2}$. Cultures were maintained in 24-well plates containing $600 \mu \mathrm{L}$ of medium at $37^{\circ} \mathrm{C}$ and $5 \% \mathrm{CO}_{2}$ in a humidified atmosphere. Culture medium was based on Neurobasal (NB; GIBCO) containing B27 supplement, transferrin, glutamine (all Sigma-Aldrich), and PSN antibiotic mixture (GIBCO). Medium changes were not performed.

\section{siRNA preparation}

Anti-EGFP siRNA for uptake studies was chemically synthesized by Biospring and $\mathrm{Cy} 3$ conjugated at the $5^{\prime}$ end. Oligonucleotides were freshly annealed before use in annealing buffer containing $100 \mathrm{mM}$ potassium acetate, $30 \mathrm{mM}$ Hepes- $\mathrm{KOH}$ ( $\mathrm{pH} 7.4$ ), and $2 \mathrm{mM}$ magnesium acetate (all Fluka). Nonlabeled anti-EGFP siRNA for silencing experiments was generated using the Silencer SiRNA Construction Kit (Ambion). The siRNA yield was quantified by spectrophotometry for each individual siRNA preparation. The following sequence was used for both siRNA preparations: anti-EGFP-sense 5'-GACGUAAACGGCCACAAGU UC-3', anti-EGFP-antisense 5'-ACUUGUGGCCGUUUACGUC GC-3'. Anti-MAP2 siRNA was synthesized by QIAGEN according to a published sequence containing anti-MAP2-sense 5'CGAGAGGAAAGACGAAGGAUU- $3^{\prime}$ and anti-MAP2-antisense 5' -UCCUUCGUCUUUCCUCUCGUG-3' (Krichevsky and Kosik 2002). Lyophilized siRNA was reconstituted following the manufacturer's instructions.

\section{Preparation of AVEs}

For the preparation of AVEs, 1,2-dioleoyl-sn-glycero-3-(phosphoL-serine) (DOPS), 1,2 dioleoyl-sn-Glycero-3-Phosphoethanolamine (DOPE) (both from Avanti Polar Lipids, Inc.) and Cholesterol (Calbiochem) were used. Lipids were used as stock solutions in Chloroform and kept for storage at $-20^{\circ} \mathrm{C}$. 


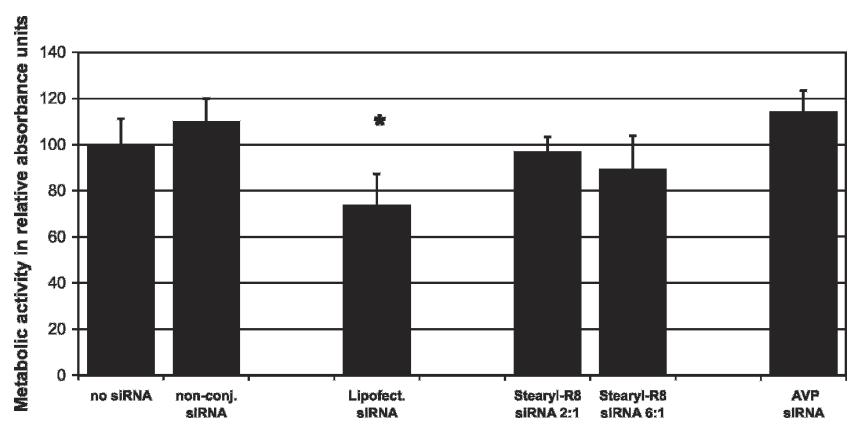

FIGURE 7. Toxicity of siRNA transfection reagents. Metabolic activity assessed by WST1 assay indicating functional integrity of the neuronal culture after application of siRNA without transfection reagents, and siRNA complexed with Lipofectamine, Stearyl-R8, or AVP $24 \mathrm{~h}$ after transfection. Results are given as relative absorption units compared with nontransfected control cultures.

For the preparation of AVE-DOPE (composition DOPS:DOPE: Chol 1:1:1) lipids were dissolved in chloroform and dried into a thin film in a rotating $100-\mathrm{mL}$ glass vessel warmed by a water bath at $40^{\circ} \mathrm{C}$ under gentle vacuum. Residual chloroform was removed by vacuum desiccation for $1 \mathrm{~h}$. The lipid film was hydrated by the addition of $1 \mathrm{~mL}$ of $40^{\circ} \mathrm{C}$ warm sterile Tris buffer $(10 \mathrm{mM}$ at $\mathrm{pH} 7.4)$, resulting in a total lipid concentration of $10 \mathrm{mM}$. The multilamellar liposome suspension was sonicated in a ultrasonic bath for $1 \mathrm{~h}$. Final preparation of the small unilamellar liposomes was done by extrusion through polycarbonate membrane filters with a pore size of $50 \mathrm{~nm}$ in a Liposofast (Avestin). Liposome size and size distribution were checked by photon correlation spectroscopy (Zetasizer Nano ZS, Malvern Instruments). Liposomes were sterile filtered $(220 \mathrm{~nm})$.

\section{siRNA application}

For transfection and targeting of EGFP-expression, siRNA was complexed with Lipofectamine 2000 (Invitrogen), Stearyl-R8, or PEI and AVEs in order to obtain AVP. All transfections were performed on day 3 in vitro (DIV 3), unless otherwise stated. Cell cultures were fixed and processed for immunocytochemistry on DIV 4 for endosomal stainings and on DIV 9 for MAP2B staining.

\section{Lipofectamine 2000}

According to the manufacturer's instructions, per well of a 24-well culture plate, $1 \mu \mathrm{L}$ of Lipofectamine 2000 was diluted in $50 \mu \mathrm{L}$ of Neurobasal medium without supplements at room temperature and 5 min later was combined with 10 pmol of siRNA in $50 \mu \mathrm{L}$ of Neurobasal medium without supplements. Incubation was continued for $20 \mathrm{~min}$ at room temperature, and the mixture was applied to culture wells, resulting in a final siRNA concentration of $16 \mathrm{nM}$.

\section{Stearyl-R8}

Transfections were performed according to an adapted protocol from DNA transfection experiments (Futaki et al. 2001a). Stearyl-R8 was chemically synthesized by SynPep. Transfection was carried out using different cation:anion charge ratios of a Stearyl-R8:siRNA mixture (data not shown). A Stearyl-R8:siRNA ratio of 2:1 yielded best results and was chosen for all further experiments. Per well of a 24-well culture plate, $1.5 \mu \mathrm{L}$ of Stearyl-R8 was diluted in $50 \mu \mathrm{L}$ of Neurobasal medium without supplements and combined with 10 pmol of siRNA in $50 \mu \mathrm{L}$ of Neurobasal medium without supplements after $5 \mathrm{~min}$ incubation at room temperature. Incubation was continued for $20 \mathrm{~min}$ at room temperature and the mixture was applied to the culture wells, resulting in a final siRNA concentration of $16 \mathrm{nM}$.

\section{$A \vee P$}

Transfection protocols were adapted from DNA transfection experiments (Nahde et al. 2001). A total of $0.3 \mu \mathrm{g}$ of low molecular weight, branched polyethyleneimine (PEI; Lupasol G100, BASF) was added in aqueous solution (adjusted to $\mathrm{pH} 7.4$ and sterile filtered) to 10 pmol of siRNA, resulting in a ratio of polyethyleneimine nitrogen:siRNA of 21:1. Lower ratios resulted in reduced transduction, while higher ratios had only marginal impact in experiments with plasmid DNA (data not shown). Complexation was performed by pipetting up and down and a further incubation for $15 \mathrm{~min}$. Next, an AVE-suspension containing $0.66 \mathrm{nmol}$ of lipid was added to $10 \mathrm{pmol}$ of siRNA, precomplexed with PEI, and gently mixed by pipetting up and down in an eppendorf tube. The formulation was continued for $20 \mathrm{~min}$ at room temperature, yielding AVPs ready for transfection. For siRNA application, experiments without cationic lipids, $10 \mathrm{pmol}$ of "naked" siRNA per well was added directly to the cell suspension after preparation. Cells were plated as described, resulting in a final siRNA concentration of $16 \mathrm{nM}$.

For cell viability experiments without siRNA, equal amounts of transfection reagents were used as mentioned above.

\section{DNA transfection and RNAi}

Freshly dissociated hippocampal neurons were DNA transfected by electroporation using the Amaxa Nucleofector (Amaxa biosystems). A total of $2.0 \times 10^{6}$ cells were resuspended in nucleofection buffer, and $3 \mu \mathrm{g}$ of DNA plasmid (pU1.EGFP) were added according to the manufacturer's instructions. Electroporation was performed using program O-05 (Gresch et al. 2004) and cells were cultured as described above. Transfection of anti-EGFP siRNA with transfection reagents was done on DIV 3. EGFP-positive neurons were counted in five random ocular fields $48 \mathrm{~h}$ after siRNA transfection using a Zeiss-Axioplan fluorescence microscope (Zeiss).

\section{Immunocytochemistry}

Cultures were fixed with formaldehyde (4\%, $10 \mathrm{~min})$, unspecific binding was blocked with normal goat serum (NGS; 10\% in PBS, $10 \mathrm{~min}$ ), and the primary antibody (anti-Lamp1, 1:250, kind gift of Dr. M. Sereda, Department of Neurogenetics, Max Planck Institut of Experimental Medicine, Göttingen) was applied in blocking solution overnight. Secondary Cy2-coupled anti-rat antibody (1:300; Jackson Labs) was applied for $45 \mathrm{~min}$ at $37^{\circ} \mathrm{C}$, and cultures were nuclear stained with DAPI (1:1000, $10 \mathrm{~min}$; Sigma-Aldrich) and mounted in Moviol (Hoechst) on glass slides. A Leica confocal laser-scanning system with a Krypton/Argon laser equipped with a Leica inverted microscope and a $40 \mathrm{X}$ objective was used (Leica).

For detection of endogenous MAP2B, hippocampal neuron cultures were processed as detailed above. Here, the primary antiMAP2B (1:400, BD Biosciences) and the secondary Cy2-coupled antimouse antibody (1:300; Jackson Labs) were used. Additionally, 
rhodamine-labeled phalloidin (1:400, Molecular Probes) was applied together with the secondary anti-mouse antibody to visualize f-actin.

\section{Western blotting}

Hippocampal neuron lysates were prepared after $7 \mathrm{~d}$ of siRNA treatment using a lysis buffer containing $10 \mathrm{mM}$ HEPES ( $\mathrm{pH}$ 7.2), $142 \mathrm{mM} \mathrm{KCl}, 5 \mathrm{mM} \mathrm{MgCl} 2,1 \mathrm{mM}$ EGTA, and 1\% IGEPAL plus protease inhibitors ("Complete tablets," Roche). Protein quantification was determined using the bicinchoninic acid assay (Pierce). Then, $20 \mu \mathrm{g}$ of protein from each sample was resolved by sodium dodecyl sulfate-polyacrylamide gel electrophoresis and transferred to polyvinylidene difluoride membranes. The membranes were blocked for $1 \mathrm{~h}$ at room temperature in a blocking solution containing 5\% BSA, $0.1 \%$ Tween-20, and Tris-buffered saline $(50 \mathrm{mM}$ Tris-Hcl at $\mathrm{pH}$ 7.6, $150 \mathrm{mM} \mathrm{NaCl}$ ). After blocking, the membranes were incubated overnight at $4^{\circ} \mathrm{C}$ with the mouse anti-MAP2B (1:1000, $\mathrm{BD}$ Biosciences) and mouse anti- $\beta$ Tubulin (1:2000, Sigma) antibodies diluted in blocking solution. The membranes were washed three times with $0.1 \%$ Tween-20 in Tris-buffered saline (15 min each) and incubated with a horseradish peroxidase-conjugated goat anti-mouse IgG antibody diluted in blocking solution (1:2000, Sigma). The membranes were washed with Tris-buffered saline as described before and then developed for analysis using a chemiluminesence method. Images were recorded and analyzed with the Quantity One Image Analysis Software (Bio-Rad).

\section{Assessment of metabolic activity}

Overall metabolic activity in culture was determined using the WST1 assay (Roche) according to the manufacturer's instructions. Briefly, WST1 reagent was added 1:10 to the culture medium and incubated for $2 \mathrm{~h}$ at $37^{\circ} \mathrm{C}$. Absorbance was measured at 450 against $620-\mathrm{nm}$ reference wavelength using an ELISA reader (Tecan).

\section{Statistics}

Data obtained in toxicity assays and cell counts are given as means \pm SD. Intergroup differences were considered significant at ${ }^{\star} p<0.05$, ${ }^{* *} p<0.01$, and ${ }^{* * *} p<0.001$ according to one-way ANOVA.

\section{ACKNOWLEDGMENTS}

We thank Dr. M. Sereda, Department of Neurogenetics, Max Planck Institut of Experimental Medicine, Göttingen, for kindly providing the Lamp1 antibody; Dr. Ch. Stadelmann, Institute of Neuropathology, University of Göttingen, for kindly providing the secondary Cy2-coupled anti-rat antibody; Alexandra Marten and Ulrike Schöll for excellent technical assistance; Ajit Singh Dhaunchak and Asparuh Iliev for help with confocal imaging; and Katrin Meuer for help with time-lapse microscopy. Funded by Deutsche Forschungsgemeinschaft through the DFG-Research Center for Molecular Physiology of the Brain.

Received October 5, 2005; accepted March 27, 2006.

\section{REFERENCES}

da Cruz, M.T., Simoes, S., and de Lima, M.C. 2004. Improving lipoplex-mediated gene transfer into C6 glioma cells and primary neurons. Exp. Neurol. 187: 65-75.
Dalby, B., Cates, S., Harris, A., Ohki, E.C., Tilkins, M.L., Price, P.J., and Ciccarone, V.C. 2004. Advanced transfection with Lipofectamine 2000 reagent: Primary neurons, siRNA, and high-throughput applications. Methods 33: 95-103.

Davidson, T.J., Harel, S., Arboleda, V.A., Prunell, G.F., Shelanski, M.L., Greene, L.A., and Troy, C.M. 2004. Highly efficient small interfering RNA delivery to primary mammalian neurons induces MicroRNA-like effects before mRNA degradation. J. Neurosci. 24: 10040-10046.

Futaki, S., Ohashi, W., Suzuki, T., Niwa, M., Tanaka, S., Ueda, K., Harashima, H., and Sugiura, Y. 2001a. Stearylated arginine-rich peptides: A new class of transfection systems. Bioconjug. Chem. 12: 1005-1011.

Futaki, S., Suzuki, T., Ohashi, W., Yagami, T., Tanaka, S., Ueda, K., and Sugiura, Y. 2001b. Arginine-rich peptides. An abundant source of membrane-permeable peptides having potential as carriers for intracellular protein delivery. J. Biol. Chem. 276: 5836-5840.

Gresch, O., Engel, F.B., Nesic, D., Tran, T.T., England, H.M., Hickman, E.S., Korner, I., Gan, L., Chen, S., Castro-Obregon, S., et al. 2004. New non-viral method for gene transfer into primary cells. Methods 33: 151-163.

Kiefer, K., Clement, J., Garidel, P., and Peschka-Suss, R. 2004. Transfection efficiency and cytotoxicity of nonviral gene transfer reagents in human smooth muscle and endothelial cells. Pharm. Res. 21: 1009-1017.

Krichevsky, A.M. and Kosik, K.S. 2002. RNAi functions in cultured mammalian neurons. Proc. Natl. Acad. Sci. 99: 11926-11929.

Lappalainen, K., Jaaskelainen, I., Syrjanen, K., Urtti, A., and Syrjanen, S. 1994. Comparison of cell proliferation and toxicity assays using two cationic liposomes. Pharm. Res. 11: 1127-1131.

Leng, Q., Scaria, P., Zhu, J., Ambulos, N., Campbell, P., and Mixson, A.J. 2005. Highly branched HK peptides are effective carriers of siRNA. J. Gene Med. 7: 977-986.

Lingor, P., Michel, U., Scholl, U., Bahr, M., and Kugler, S. 2004. Transfection of "naked" siRNA results in endosomal uptake and metabolic impairment in cultured neurons. Biochem. Biophys. Res. Commun. 315: 1126-1133.

Luo, D. and Saltzman, W.M. 2000. Synthetic DNA delivery systems. Nat. Biotechnol. 18: 33-37.

Maurer, N., Mori, A., Palmer, L., Monck, M.A., Mok, K.W., Mui, B., Akhong, Q.F., and Cullis, P.R. 1999. Lipid-based systems for the intracellular delivery of genetic drugs. Mol. Membr. Biol. 16: 129-140.

Muller, K., Nahde, T., Fahr, A., Muller, R., and Brusselbach, S. 2001. Highly efficient transduction of endothelial cells by targeted artificial virus-like particles. Cancer Gene Ther. 8: 107-117.

Muratovska, A. and Eccles, M.R. 2004. Conjugate for efficient delivery of short interfering RNA (siRNA) into mammalian cells. FEBS Lett. 558: 63-68.

Nahde, T., Muller, K., Fahr, A., Muller, R., and Brusselbach, S. 2001. Combined transductional and transcriptional targeting of melanoma cells by artificial virus-like particles. J. Gene Med. 3: 353361.

Patil, S.D., Rhodes, D.G., and Burgess, D.J. 2004. Anionic liposomal delivery system for DNA transfection. AAPS J. 6: e29.

Sledz, C.A., Holko, M., de Veer, M.J., Silverman, R.H., and Williams, B.R. 2003. Activation of the interferon system by short-interfering RNAs. Nat. Cell Biol. 5: 834-839.

Sontheimer, E.J. 2005. Assembly and function of RNA silencing complexes. Nat. Rev. Mol. Cell Biol. 6: 127-138.

Vistica, D.T., Skehan, P., Scudiero, D., Monks, A., Pittman, A., and Boyd, M.R. 1991. Tetrazolium-based assays for cellular viability: A critical examination of selected parameters affecting formazan production. Cancer Res. 51: 2515-2520.

Welz, C., Neuhuber, W., Schreier, H., Metzler, M., Repp, R., Rascher, W., and Fahr, A. 2000. Nuclear transport of oligonucleotides in HepG2-cells mediated by protamine sulfate and negatively charged liposomes. Pharm. Res. 17: 1206-1211.

Zeng, Y. and Cullen, B.R. 2002. RNA interference in human cells is restricted to the cytoplasm. RNA 8: 855-860. 

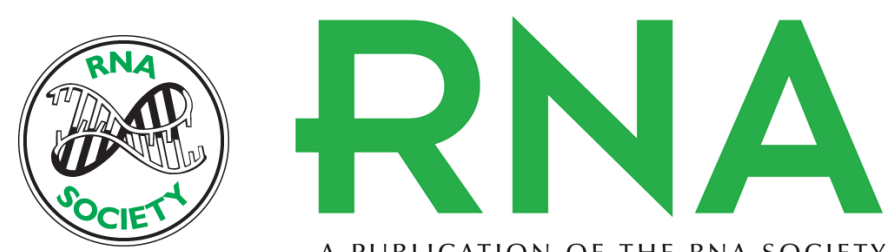

A PUBLICATION OF THE RNA SOCIETY

\section{Stearylated octaarginine and artificial virus-like particles for transfection of siRNA into primary rat neurons}

Lars Tönges, Paul Lingor, Roman Egle, et al.

RNA 2006 12: 1431-1438

References This article cites 21 articles, 5 of which can be accessed free at:

http://rnajournal.cshlp.org/content/12/7/1431.full.html\#ref-list-1

License

Email Alerting Receive free email alerts when new articles cite this article - sign up in the box at the Service top right corner of the article or click here.

To subscribe to RNA go to:

http://rnajournal.cshlp.org/subscriptions 